\title{
Distribución espacial y temporal del cangrejo Callinectes sapidus (Decapoda: Portunidae) en la Bahía de Chetumal, Quintana Roo, México
}

\author{
Héctor J. Ortiz-León ${ }^{1}$, Alberto de Jesús-Navarrete ${ }^{2} \&$ Eloy Sosa Cordero $^{2}$ \\ 1 Instituto Tecnológico de Chetumal, A. P. 267, Chetumal, Quintana Roo, México, C. P. 77013; oilh@yahoo.com.mx \\ 2 El Colegio de la Frontera Sur, Unidad Chetumal. A. P. 424, Chetumal, Quintana Roo, México, C. P. 77900; alberto@, \\ ecosur-qroo.mx, esosa@ecosur-qroo.mx
}

Recibido 13-II-2003. Corregido 27-VI-2006. Aceptado 15-XII-2006.

\begin{abstract}
Temporal and spatial distribution of the crab Callinectes sapidus (Decapoda: Portunidae) in Chetumal Bay, Quintana Roo, Mexico. In order to determine temporal and spatial distribution patterns of Callinectes sapidus, samplings were carried out during the cold-front (January-February), dry (May-June) and rainy (August-September, 2002) climatic seasons, in 30 sampling stations of Chetumal Bay, grouped in sectors A (14 stations), B (eight stations) and C (eight stations). In each sampling station crabs were collected from two transects parallel to the coast, each with three traps, separated by $30 \mathrm{~m}$. Sediments were calcareous coarse and medium sand, white or lightly gray. A total of 1031 specimens were collected. CPEU (Capture Per Effort Unit) differed spatially and temporally. Highest CPEU was found in sector C with 1.3 ind trap $^{-1}$, and in the rainy season with 1.1 ind trap $^{-1}$. Population was predominantly composed of male individuals. The male:female ratio was 15:1. Males and adults (group II) CPEU was significant different between sectors and climatic seasons. Both males and adults (group II) had a greater CPEU in sector C (1.2 ind $\left.\cdot \operatorname{trap}^{-1}\right)$ and in the rainy season (1.1 ind $\cdot \operatorname{trap}^{-1}$ ). Abundance of female and juvenile individuals (group I) was low during the sampling period whereas group 0 juvenile individuals were not found. A greater relative frequency between sectors and climatic seasons were observed in 130-139 mm and 140-149 mm size interval (CW). C. sapidus occurred on sandy sediments in Chetumal Bay. Pearson product moment correlations exhibited significant relationships between CPEU and temperature, salinity and dissolved oxygen. In Chetumal Bay, the spatial and temporal distribution of C. sapidus can be related to salinity, temperature, habitat quality, food availability, recruitment and reproduction events of individuals. Rev. Biol. Trop. 55 (1): 235-245. Epub 2007 March. 31.
\end{abstract}

Key words: Caribbean, Chetumal Bay, blue crab, Callinectes sapidus, distribution.

El género Callinectes está confinado a las aguas costeras someras y exhibe una radiación hacia el norte y el sur, desde el centro del Atlántico Neotropical, así como en el Pacífico Tropical Oriental y a lo largo del oeste de África Tropical (Williams 1974). En total, 11 especies se encuentran en el Atlántico y tres especies en el Pacífico. La salinidad es el factor que ejerce mayor influencia en la distribución de Callinectes spp. del Caribe, dentro y entre las islas (Norse 1972, 1977, 1978). Sus patrones de distribución espacial parecen estar más relacionados con las características particulares del hábitat, como el sedimento, extensión del estuario y la cercanía a los manglares, que les proveen refugio (Buchanan y Stoner 1988).

La jaiba azul, Callinectes sapidus Rathbun, 1896, se distribuye desde Nueva Escocia, Canadá hasta río de la Plata, Argentina e incluye el Mar Caribe y el Golfo de México (Williams 1974). Según Norse (1978) C. sapi$d u s$ y $C$. bocourti son mucho más tolerantes a bajas salinidades que $C$. danae y $C$. ornatus. Grandes fluctuaciones anuales en la abundancia son características de las poblaciones de C. sapidus (Hines et al. 1987). En una laguna costera donde los cambios entre el verano y el 
invierno son de $10^{\circ} \mathrm{C}$, es posible que las necesidades de energía de $C$. sapidus, aumenten en relación conl incremento de la temperatura (Rosas y Sánchez 1994). El apareamiento se encuentra en regiones de baja salinidad, de donde las hembras maduras migran para desovar a las áreas de mayor salinidad (Churchill 1919, Van Engel 1958). Por ello, la jaiba azul C. sapidus utiliza tanto el ecosistema oceánico como el estuarino durante su ciclo de vida.

El sedimento en el medio acuático es de gran importancia. Las aguas estuarinas someras con sedimentos blandos de detritus, fango o conchas-fango, son el hábitat óptimo para las jaibas pequeñas, mientras que las grandes prefieren aguas salobres profundas con sedimentos duros (Adkins 1972).

El contenido de materia orgánica es dinámico y puede cambiar con base en la concentración en la columna de agua, y dadas las características diagenéticas y geológicas de cada región, tales como los procesos de degradación bacteriana, actividad de los organismos bénticos y tasas de sedimentación, se proveen de materiales remineralizados a los consumidores primarios y alimentación para los consumidores bénticos, algunos de ellos de importancia comercial (De la Lanza 1986, De Jesús-Navarrete et al. 2000), como las jaibas.

Aún cuando existen investigaciones sobre la biología, distribución, reproducción y manejo pesquero de las jaibas, especialmente de $C$. sapidus en Estados Unidos (Churchill 1919, Van Engel 1958, Adkins 1972, Williams 1974, Millikin y Williams 1984, Hines et al. 1987, Schaffner y Díaz 1988, Rothschild et al. 1992, Guillory et al. 1996, Tankersley et al. 1998), esta información no puede ser generalizada a otras regiones, debido a diferencias geomorfológicas, patrones de salinidad y circulación, que influyen en la distribución y abundancia de los organismos en ambientes estuarinos.

Para la región del Caribe se han publicado trabajos, de aprovechamiento pesquero, relaciones morfométricas, alimentación, fecundidad, patrones de distribución y migración larvaria, de especies de Callinectes (Norse 1972, 1977, 1978, Moncada y Gómez 1980,
Rodríguez 1982, Buchanan y Stoner 1988, Criales et al. 2002).

En México, la pesca de la jaiba azul C. sapidus es una actividad importante, y se han realizado estudios sobre su biología, distribución, ecología, etología, fisiología y pesquerías (Román-Contreras 1986a, b, Rosas y Lázaro-Chávez 1986, García-Montes et al. 1988, Rocha-Ramírez et al. 1992, Rosas y Sánchez 1994, Granados et al. 1995, Andrade 1996, Ramos et al. 1998, Manzanero 1999). En Quintana Roo, Markham et al. (1987) informaron cinco especies de jaibas para la Bahía de la Ascensión y solamente una especie para la Bahía de Chetumal

En la Bahía de Chetumal no hay antecedentes de estudios sobre biología, ecología y pesquerías de esta especie; por esa razón, el objetivo de esta investigación fue determinar los patrones espacio-temporales de distribución y abundancia por sexo y estadio de madurez sexual de la jaiba azul C. sapidus en la Bahía de Chetumal, Quintana Roo.

\section{MATERIALES Y MÉTODOS}

Área de estudio: El estudio se realizó en la Bahía de Chetumal, (18 $11^{\circ}-18^{\circ} 52^{\prime} \mathrm{N}$, $\left.87^{\circ} 51^{\prime}-88^{\circ} 23^{\prime} \mathrm{W}\right)$, perteneciendo a México y Belice. La Bahía es alargada, de contorno irregular, una extensión de $67 \mathrm{~km}$ y una anchura máxima de $20 \mathrm{~km}$, con una superficie de 1098 $\mathrm{km} 2$. Es considerada un estuario con aportes importantes de agua dulce, principalmente del río Hondo y pequeños manantiales de la laguna Guerrero (Gasca y Castellanos 1993). La vegetación predominante es el manglar y con menor cobertura macroalgas y fanerógamas acuáticas (Morales-Vela et al. 1996, Olivera-Gómez 2002). Debido a sus características físicas, la renovación y la circulación del agua es lenta (Hernández y Pérez 1991).

Las jaibas se recolecciónron de enero a septiembre de 2002. Se realizaron tres muestreos, al final de un mes y principios del otro, con una duración de nueve días cada uno, durante tres temporadas climáticas: "nortes" 
(enero-febrero), secas (mayo-junio) y lluvias (agosto-septiembre). El área de estudio se dividió en celdas de 1 min geográfico por lado (Morales-Vela et al. 1996), agrupándose en áreas de 12 × 24 celdas, quedando dividida en tres sectores, los cuales siguen un patrón de sur a norte y de este a oeste, y cubrieron con cierta homogeneidad las costas de ambos lados y parte central de la Bahía de Chetumal. Se eligieron al azar 30 estaciones de muestreo, ubicando 14 estaciones en el sector A, ocho en el sector B y ocho en el sector C (Fig. 1). Los sitios de muestreo se ubicaron con un geoposicionador Garmin ${ }^{\circledR}$ 45. El recorrido entre los puntos de muestreo se realizó a bordo de una lancha de 25 pies de eslora con un motor fuera de borda de $40 \mathrm{HP}$.

Los factores ambientales se registraron simultáneamente con el muestreo biológico. La temperatura, salinidad y oxígeno disuelto se midieron con un equipo YSI ${ }^{\circledR}$ modelo 85 . La profundidad se midió con una sondaleza marcada a intervalos de $10 \mathrm{~cm}$. En cada sitio se tomaron muestras de sedimentos con un tubo de PVC de $5 \mathrm{~cm}$ de diámetro y $50 \mathrm{~cm}$ de largo, introduciéndolo $10 \mathrm{~cm}$ en el sustrato. La materia orgánica en los sedimentos se determinó mediante la técnica de Dean (1974). El sedimento libre de materia orgánica se tamizó en un agitador para tamices marca SIE/E Shaker ${ }^{\circledR}$, modelo RX-86 por $15 \mathrm{~min}$, utilizando una serie de tamices de: $1.68,1.41,1.19,1.0,0.84,0.5,0.35,0.25,0.177$, $0.149,0.125,0.105$, y $0.074 \mathrm{~mm}$ de abertura de malla. Las clases de tamaño se clasificaron con la escala de Wenworth (Folk 1969).

Las jaibas se recolecciónron utilizando dos tipos de trampas cangrejeras de estructura de alambrón. Las primeras, forradas de tela de alambre con una abertura de malla de $2.54 \mathrm{~cm}$, con dimensiones de 45 x 45 x $40 \mathrm{~cm}$; las segundas, forradas de malla alquitranada de $2.54 \mathrm{~cm}$ de luz de malla y con dimensiones de 100 x 50 x $30 \mathrm{~cm}$. En cada sitio de muestreo se ubicaron dos transectos paralelos a la costa, colocando tres trampas en cada transecto, separadas $30 \mathrm{~m}$ una de otra. Se utilizó bagre como carnada, fijo al centro de la trampa. Las trampas fueron colocadas en cada sector, a partir de las 09:00 h,

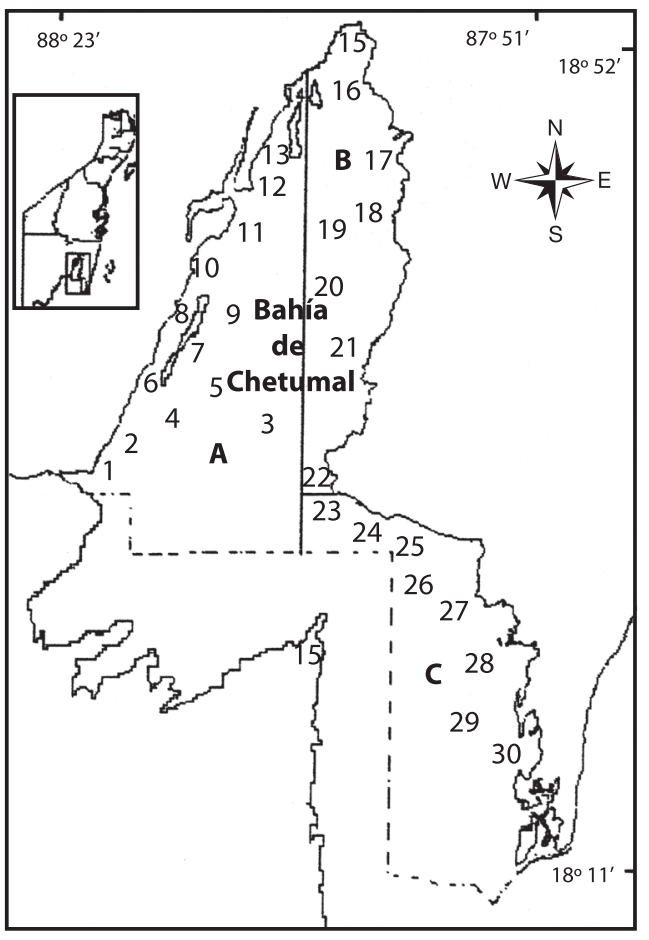

Fig. 1. Ubicación de las 30 estaciones de muestreo en tres sectores de la Bahía de Chetumal. Sector A: 1) Bellavista 2) UQROO 3) Bahía Centro 4) Calderitas 5) Calderitas Exterior 6) Tamalcab Sur 7) Tamalcab Centro 8) Tamalcab Norte 9) Tamalcab-2 Hermanos 10) La Saladora 11) Punta Lagarto 12) La Barra 13) CayoVenado Sur 14) CayoVenado-Norte. Sector B: 15) Río Crick 16) Punta Gorda-Cayo Violín 17) Siete Esteros 18) Punta Nicte-Chón 19) La Barra-Punta Nicte-Chón 20) Punta Nicte-Chón-Dos Hermanos 21) Dos Hermanos 22) Punta Flor-Punta Calentura. Sector C: 23) Punta Calentura 24) Cayo Cazón 25) Punta Jazz 26) Punta Mainada-Punta Jazz 27) Punta Mainada 28) Siete Mogotes 29) Mala Noche 30) La Aguada.

Fig. 1. Location of the 30 sampling stations in three sectors of Chetumal Bay.

extrayendo su contenido al día siguiente y repitiendo una vez más esta operación. Las jaibas fueron identificadas, sexadas y medidas, siendo liberadas posteriormente. La identificación se realizó de acuerdo con Williams (1974). El sexo se determinó por la forma del abdomen, según lo señalado por Millikin y Williams (1984). La anchura del caparazón (AC), que comprende las espinas laterales, se midió con 
un vernier, con precisión de $1 \mathrm{~mm}$. El estadio de madurez sexual se determinó de acuerdo con los grupos de talla (edad) por sexo de Rothschild et al. (1992). Se reconocieron los grupos 0: $\leq 49$ mm AC y I: $49<x \leq 120 \mathrm{~mm} \mathrm{AC,} \mathrm{como} \mathrm{jóvenes}$ (machos y hembras inmaduras), y II: $\geq 120$ $\mathrm{mm} \mathrm{AC}$, como adultos (individuos maduros de ambos sexos). El índice de abundancia se representó como captura por unidad de esfuerzo (CPUE), expresado en ind trampa ${ }^{-1}$.

Las diferencias entre los valores promedios de los factores ambientales, CPUE y la abundancia de machos, hembras, adultos y jóvenes entre sectores y temporadas climáticas fueron comparadas con ANOVA de dos-vías. La prueba de ámbito múltiple de Tukey HSD se utilizó para comparaciones múltiples donde hubo diferencias significativas del ANOVA. En todos los casos se revisó el cumplimiento de los requisitos del ANOVA. Se graficó la frecuencia relativa de C. sapidus por sectores y por temporadas climática, empleando intervalos de talla de $10 \mathrm{~mm}(\mathrm{AC})$. La correlación producto momento de Pearson se empleó para evaluar las relaciones entre la abundancia de $C$. sapidus y los factores ambientales.

\section{RESULTADOS}

Se recolectaron un total de 1031 ejemplares de C. sapidus. La CPUE promedio en las estaciones de muestreo varió entre 0.3 ind trampa $^{-1}$ y 1.8 ind trampa $^{-1}$ los valores menores se encontraron en las estaciones Cayo Venado Norte, Cayo Venado Sur, La BarraPunta Nicte-Chón y Punta Nicte-Chón- Dos Hermanos (sector B) y el valor mayor en la estación La Aguada (sector C). La CPUE difirió significativamente espacialmente, entre sectores $(\mathrm{F}=13.04, \mathrm{p}<0.05)$ con la más baja CPUE promedio en el sector $\mathrm{B}\left(0.6\right.$ ind trampa $\left.{ }^{-1}\right)$ que en los sectores A y C (1.0 y 1.3 ind $\cdot$ trampa $^{-1}$, respectivamente), lo mismo que entre temporadas $(\mathrm{F}=5.42, \mathrm{p}<0.05)$, siendo en "nortes" cuando se registró la menor CPUE (0.7 ind·trampa $\left.{ }^{-1}\right)$, que en las temporadas de secas y lluvias (1.0 y 1.1 ind $\cdot$ trampa ${ }^{-1}$, respectivamente). De manera espacio-temporal, durante la temporada de "nortes", cuando la temperatura fue menor, también se registró la menor CPUE, con 0.4 ind trampa ${ }^{-1}$ en el sector B (Fig. 2).

La población de C. sapidus estuvo compuesta predominantemente por machos. $\mathrm{La}$ relación macho:hembra fue 15:1. Del promedio general de la CPUE por sexo, 0.9 ind trampa $^{-1}$ correspondió a machos y 0.1 ind trampa $^{-1}$ a hembras. La CPUE de machos fue significativamente diferente entre sectores $(\mathrm{F}=14.42$, $\mathrm{p}<0.05)$, siendo la CPUE más baja en el sector $\mathrm{B}$ (0.5 ind trampa $\left.{ }^{-1}\right)$, que en los sectores $\mathrm{A}$ y C (0.9 y 1.2 ind $\cdot$ trampa $^{-1}$, respectivamente). También presentó diferencias significativas entre temporadas $(\mathrm{F}=6.41, \mathrm{p}<0.05)$, siendo la menor CPUE ( 0.6 ind $\cdot$ trampa $\left.^{-1}\right)$ durante la temporada de "nortes" y mayor en secas y lluvias (1.0 y 1.1 ind trampa ${ }^{-1}$, respectivamente). En términos espacio-temporales, los machos de $C$. sapidus presentaron la menor CPUE en el sector $\mathrm{B}$, con 0.3 ind trampa ${ }^{-1}$ durante la temporada de "nortes", mientras que en el sector $\mathrm{C}$ durante la temporada de secas se registró la mayor CPUE con 1.4 ind trampa ${ }^{-1}$ (Fig. 3A). La CPUE de hembras fue escasa durante todo el período de muestreo. De la CPUE de hembras, 0.03 ind trampa ${ }^{-1}$ correspondió

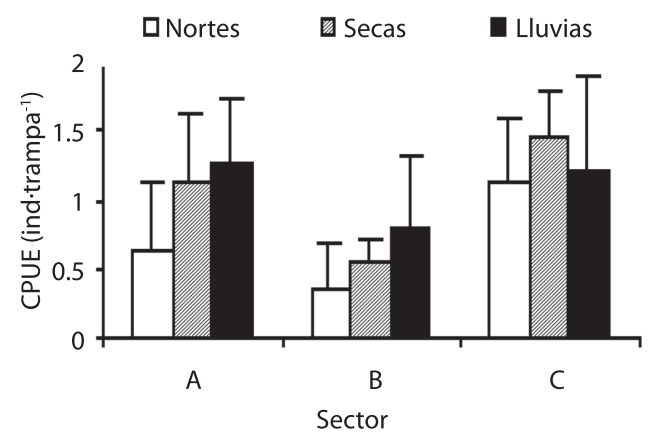

Fig. 2. CPUE (ind trampa ${ }^{-1}$ ) promedio de C. sapidus en la Bahía de Chetumal durante las estaciones climáticas de "nortes", secas y lluvias. Las barras representan los valores promedio de la CPUE $( \pm 1 \mathrm{DE})$ registrados en los tres sectores de la Bahía de Chetumal.

Fig. 2. Mean CPEU (ind trap $^{-1}$ ) of $C$. sapidus in Chetumal Bay during the cold front, dry and rainy climatic seasons. Bars represent mean CPEU $( \pm 1 \mathrm{SE})$ values registered in three sectors of Chetumal Bay. 

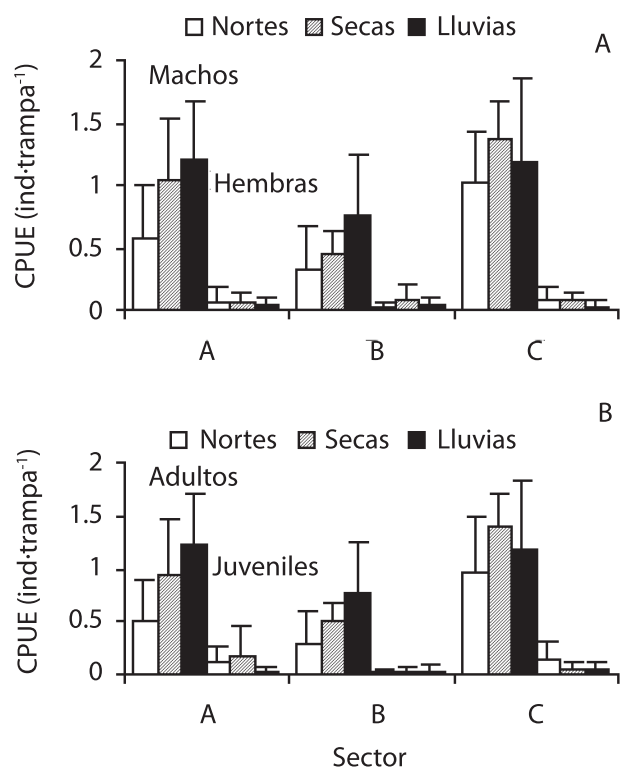

Fig. 3. CPUE (ind $\cdot$ trampa $^{-1}$ ) promedio de C. sapidus (A.) por sexo y (B.) por estadio de madurez en la Bahía de Chetumal. Las barras representan los valores promedio de la CPUE $( \pm 1 \mathrm{DE})$ registrados en los tres sectores de la Bahía de Chetumal, durante las estaciones climáticas de "nortes", secas y lluvias.

Fig.3. Mean CPEU (indtrap ${ }^{-1}$ ) of C. sapidus (A.) by sex and (B.) by maturity stage in Chetumal Bay. Bars represent mean CPEU $( \pm 1 \mathrm{SE})$ values registered in three sectors of Chetumal Bay, during the cold front, dry and rainy climatic seasons.

a hembras inmaduras y 0.07 ind $\cdot \operatorname{trampa}^{-1} \mathrm{a}$ hembras maduras.

En cuanto al estadio de madurez sexual, se obtuvo un promedio general de 0.9 ind trampa${ }^{1}$ para adultos (grupo II), y 0.1 ind $\cdot$ trampa ${ }^{-}$ ${ }^{1}$ para los jóvenes (grupo I). La CPUE de adultos (grupo II), fue significativamente diferente entre sectores $(\mathrm{F}=11.93, \mathrm{p}<0.05)$, siendo la CPUE más baja en el sector $\mathrm{B}(0.5$ ind trampa $\left.^{-1}\right)$, que en los sectores A y C (0.9 y 1.2 ind trampa ${ }^{-1}$, respectivamente). También presentó diferencias significativas entre temporadas $(\mathrm{F}=7.70, \mathrm{p}<0.05)$, siendo la menor CPUE (0.6 ind trampa $\left.{ }^{-1}\right)$ durante la temporada de "nortes" y mayor en secas y lluvias (1.0 y 1.1 ind trampa ${ }^{-1}$, respectivamente). En términos espacio-temporales, los adultos de C. sapidus presentaron la menor CPUE en el sector B, con 0.3 ind trampa ${ }^{-1}$ durante la temporada de "nortes", mientras que en el sector $\mathrm{C}$ durante la temporada de secas se registró la mayor CPUE con 1.4 ind trampa $^{-1}$ (Fig. 3B). La CPUE de jóvenes del grupo 0 fue nula., mientras que los del grupo I fue escasa durante todo el período de muestreo. La mayor CPUE de jóvenes del grupo I se presentó en el sector $\mathrm{A}$, y en las temporadas climáticas de "nortes" y secas (Fig. 3B).

La mayor frecuencia relativa entre los sectores y las temporadas climáticas se observaron en los intervalos de talla (AC) de 130-139 mm y 140-149 mm (Fig. 4A, B). La correlación producto momento de Pearson, mostró relaciones significativas entre la CPUE de $C$. sapidus con la temperatura $\left(\mathrm{r}^{2}=0.37\right.$, $\mathrm{p}<0.001)$ y la salinidad $\left(\mathrm{r}^{2}=0.50, \mathrm{p}<0.001\right)$. En el sector $\mathrm{C}$, donde se registró la mayor CPUE, la salinidad mostró el mayor valor con $12.7 \pm 1.6 \%$. La mayor CPUE se presentó en profundidades promedio de $2.1 \pm 0.1 \mathrm{~m}$ y sedimentos arenosos $(>95 \%)$.
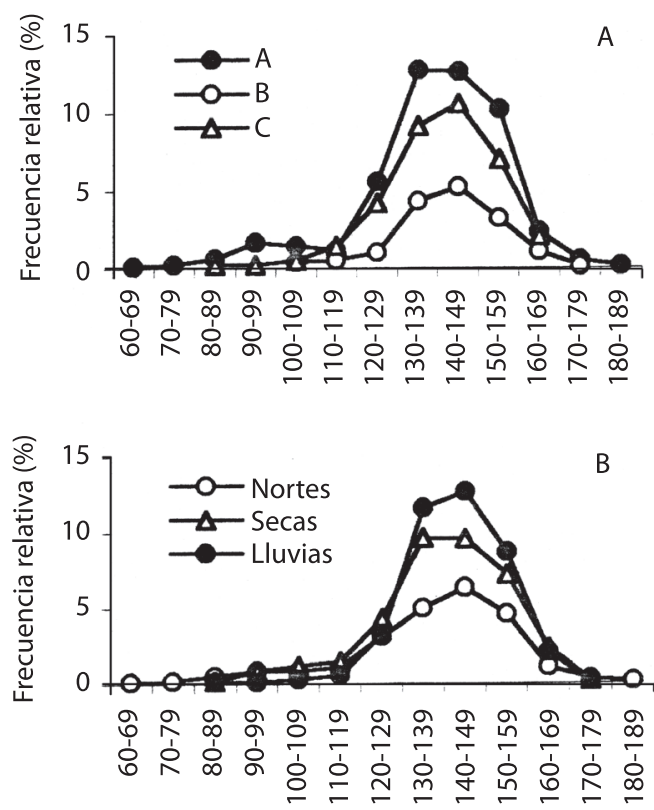

Ancho del caparazón (mm)

Fig. 4. Distribución de frecuencias de talla de C. sapidus por (A.) sector (A, B, C) y (B.) temporada climática ("nortes", secas y lluvias).

Fig. 4. Frequency distribution of $C$. sapidus by sizes (A.) sector (A, B, C) and (B.) climatic season (cold front, dry and rainy). 
La temperatura difirió significativamente entre temporadas climáticas $(\mathrm{F}=12.06$, $\mathrm{p}<0.05$ ), con la menor temperatura en la temporada de "nortes" (Cuadro 1A). La temperatura máxima de $33.1^{\circ} \mathrm{C}$ se registró en la estación Tamalcab Sur (temporada de secas) y la mínima de $22.5^{\circ} \mathrm{C}$ en la estación Punta Nicte-Chón (temporada de nortes). La salinidad difirió significativamente entre sectores $(\mathrm{F}=49.7$, $\mathrm{p}<0.05$ ), con la mayor salinidad en el sector $\mathrm{C}$, y entre temporadas $(\mathrm{F}=70.6, \mathrm{p}<0.05)$, con una variación de la salinidad de $4 \%$ entre las temporadas de nortes y lluvias (Cuadro 1A, B). La salinidad máxima de $16.8 \%$ se midió en Mala Noche, ubicada en uno de los sitios más al sur de la Bahía, y la mínima de $2.4 \%$ en la estación río Crick, en la parte más interna. $\mathrm{La}$ profundidad difirió significativamente entre sectores $(F=32.89, p<0.05)$, siendo el sector $\mathrm{C}$ (Cuadro 1B) donde la profundidad fue menor y más homogénea entre las estaciones de muestreo. La profundidad máxima de $5.4 \mathrm{~m}$ se registró en la estación Punta Nicte Chón-Dos Hermanos y la mínima de $0.5 \mathrm{~m}$ en la estación Cayo Venado Norte.
Los sedimentos fueron principalmente arenas, que variaron de muy fina a muy gruesa, de composición calcárea, blancos o ligeramente grises. Todas las estaciones presentaron más del $92 \%$ de arena; sólo las estaciones Siete Esteros y Punta Flor-Punta Calentura del Sector B, y las estaciones Cayo Cazón, Punta Mainada y Mala Noche del Sector C, tuvieron más del $5 \%$ de limos y arcillas. El porcentaje de materia orgánica varió entre $4.4 \%$ y $18.7 \%$; el valor mínimo se encontró en la estación Dos Hermanos del sector B y el máximo en la estación Siete Mogotes del sector C (Cuadro 2).

\section{DISCUSIÓN}

C. sapidus es especialmente abundante en las bahías y en las bocas de los ríos. Se ha encontrado en aguas dulces, salobres y lagunas hiperhalinas (Churchill 1919, Williams 1974). Gracias a su capacidad adaptativa, C. sapidus puede tolerar amplios intervalos de salinidad y así ocupar diversos hábitats de una laguna costera (Rosas y Lázaro-Chávez 1986, Rosas

CUADRO 1

Factores ambientales en la Bahía de Chetumal (A.) por sectores y (B.) por temporada climática

TABLE 1

Environmental factors in Chetumal Bay (A.) by sectors and (B.) by climatic season

A.

$\begin{array}{ccccc}\text { Temporada } & \text { Temperatura }\left({ }^{\circ} \mathrm{C}\right) & \text { Salinidad }(\%) & \text { Oxígeno }\left(\mathrm{mg}^{-1}\right) & \text { Profundidad }(\mathrm{m}) \\ \text { Nortes } & 25.7 \pm 1.9(\mathrm{n}=30) & 8.5 \pm 2.1(\mathrm{n}=30) & 7.5 \pm 0.9(\mathrm{n}=30) & 2.4 \pm 0.4(\mathrm{n}=30) \\ \text { Secas } & 30.9 \pm 0.3(\mathrm{n}=30) & 11.2 \pm 1.8(\mathrm{n}=30) & 6.5 \pm 0.0(\mathrm{n}=30) & 2.7 \pm 0.5(\mathrm{n}=30) \\ \text { Lluvias } & 30.4 \pm 0.9(\mathrm{n}=30) & 12.5 \pm 1.3(\mathrm{n}=30) & 5.8 \pm 0.1 \quad(\mathrm{n}=30) & 2.7 \pm 0.5(\mathrm{n}=30)\end{array}$

B.

$\begin{array}{crrrr}\text { Sector } & \text { Temperatura }\left({ }^{\circ} \mathrm{C}\right) & \text { Salinidad }(\%) & \text { Oxigeno }\left(\mathrm{mg}^{-1}\right) & \text { Profundidad }(\mathrm{m}) \\ \text { A } & 29.2 \pm 3.1(\mathrm{n}=42) & 9.8 \pm 2.1(\mathrm{n}=42) & 6.8 \pm 1.4(\mathrm{n}=42) & 2.9 \pm 0.2(\mathrm{n}=42) \\ \text { B } & 28.6 \pm 4.1(\mathrm{n}=24) & 9.7 \pm 2.4(\mathrm{n}=24) & 6.7 \pm 0.8(\mathrm{n}=24) & 2.8 \pm 0.3(\mathrm{n}=24) \\ \text { C } & 29.2 \pm 1.5(\mathrm{n}=24) & 12.7 \pm 1.6(\mathrm{n}=24) & 6.3 \pm 0.3(\mathrm{n}=24) & 2.1 \pm 0.1(\mathrm{n}=24)\end{array}$

Los valores son los promedios \pm desviación estándar en las estaciones de muestreo.

Values are means \pm standard deviation in sampling stations. 
CUADRO 2

Porcentaje de arena, limo, arcilla y materia orgánica en los sedimentos de 30 estaciones de muestreo de la Bahía de Chetumal

TABLE 2

Percentage of sand, mud, clay and organic matter content in sediments of 30 sampling sites of Chetumal Bay

\begin{tabular}{|c|c|c|c|c|c|c|c|c|}
\hline Sector & Estaciones & $\begin{array}{c}\% \text { arena } \\
\text { muy gruesa }\end{array}$ & $\begin{array}{c}\% \text { arena } \\
\text { gruesa }\end{array}$ & $\begin{array}{l}\% \text { arena } \\
\text { mediana }\end{array}$ & $\begin{array}{l}\% \text { arena } \\
\text { fina }\end{array}$ & $\begin{array}{l}\text { \%arena } \\
\text { muy fina }\end{array}$ & $\begin{array}{l}\% \text { limos } \\
\text { y arcillas }\end{array}$ & $\begin{array}{l}\% \text { materi } \\
\text { orgánica }\end{array}$ \\
\hline \multirow[t]{14}{*}{ A } & Bellavista & 29.3 & 17.6 & 19.8 & 19.2 & 9.6 & 4.4 & 10.6 \\
\hline & UQROO & 34.0 & 18.0 & 16.7 & 21.6 & 7.9 & 1.8 & 14.5 \\
\hline & Bahía Centro & 28.0 & 36.0 & 13.0 & 15.0 & 6.2 & 1.7 & 12.6 \\
\hline & Calderitas & 26.7 & 20.1 & 22.5 & 18.6 & 10.4 & 1.7 & 10.3 \\
\hline & Calderitas Exterior & 29.0 & 15.1 & 17.3 & 21.7 & 14.6 & 2.3 & 10.8 \\
\hline & Tamalcab Sur & 3.9 & 13.1 & 56.9 & 19.0 & 5.9 & 1.3 & 16.9 \\
\hline & Tamalcab Centro & 7.7 & 20.3 & 28.1 & 27.8 & 12.7 & 3.3 & 16.0 \\
\hline & Tamalcab Norte & 22.7 & 23.4 & 19.6 & 20.7 & 10.3 & 3.3 & 9.9 \\
\hline & Tamalcab-Dos Hnos. & 19.4 & 21.6 & 26.3 & 21.5 & 10.1 & 1.1 & 10.4 \\
\hline & La Saladora & 54.3 & 27.2 & 11.2 & 5.1 & 1.4 & 0.9 & 4.8 \\
\hline & Punta Lagarto & 40.3 & 17.0 & 17.5 & 15.7 & 7.6 & 2.0 & 10.5 \\
\hline & La Barra & 39.3 & 46.2 & 8.0 & 4.4 & 1.5 & 0.7 & 4.5 \\
\hline & Cayo Venado Sur & 26.6 & 20.9 & 17.1 & 20.2 & 11.2 & 4.1 & 8.7 \\
\hline & Cayo Venado Norte & 53.5 & 17.3 & 10.0 & 9.0 & 5.4 & 4.7 & 11.5 \\
\hline \multirow[t]{8}{*}{ B } & Río Crick & 51.2 & 16.6 & 11.1 & 11.5 & 6.9 & 2.6 & 11.9 \\
\hline & P. Gorda-Cayo Violín & 59.3 & 16.9 & 8.5 & 7.3 & 5.0 & 3.1 & 17.2 \\
\hline & Siete Esteros & 19.3 & 12.9 & 18.6 & 26.5 & 17.0 & 5.8 & 6.6 \\
\hline & Punta Nicte-Chón & 34.0 & 12.6 & 12.5 & 23.1 & 15.1 & 2.5 & 5.2 \\
\hline & La Barra - Nicte-Chón & 0.6 & 10.2 & 52.6 & 22.2 & 12.4 & 2.0 & 10.9 \\
\hline & Nicte-Chón-Dos Hnos. & 1.3 & 11.3 & 33.3 & 30.5 & 18.9 & 4.7 & 12.8 \\
\hline & Dos Hermanos & 15.3 & 38.3 & 34.7 & 8.3 & 2.6 & 0.7 & 4.4 \\
\hline & P. Flor -P. Calentura & 21.6 & 15.8 & 19.2 & 22.1 & 15.5 & 5.8 & 9.2 \\
\hline \multirow[t]{8}{*}{$\mathrm{C}$} & Punta Calentura & 38.7 & 26.7 & 14.3 & 10.8 & 5.9 & 3.6 & 14.9 \\
\hline & Cayo Cazón & 36.7 & 19.6 & 13.6 & 14.6 & 10.2 & 5.3 & 12.3 \\
\hline & Punta Jazz & 19.2 & 13.0 & 19.3 & 34.6 & 11.9 & 1.9 & 8.9 \\
\hline & P. Mainada-P. Jazz & 17.4 & 10.8 & 15.3 & 35.7 & 16.4 & 4.4 & 9.7 \\
\hline & Punta Mainada & 33.1 & 18.3 & 13.0 & 12.5 & 15.3 & 7.8 & 13.9 \\
\hline & Siete Mogotes & 41.2 & 21.2 & 13.5 & 11.7 & 7.8 & 4.5 & 18.7 \\
\hline & Mala Noche & 35.3 & 18.9 & 14.2 & 13.9 & 11.0 & 6.7 & 18.6 \\
\hline & La Aguada & 35.3 & 25.2 & 18.6 & 13.9 & 4.9 & 2.2 & 15.1 \\
\hline
\end{tabular}


y Sánchez 1994). En Laguna Joyuda, Puerto Rico, C. sapidus se encontró en salinidades entre $23.8 \pm 5.4 \%$ y $26.7 \pm 5.4 \%$ (Buchanan y Stoner 1988). En México, C. sapidus se ha informado en diferentes ámbitos de salinidad: 1.9-25 \%o en Veracruz (Arreguín-Sánchez 1976); 7-38 \%o en Campeche (Raz-Guzmán et al. 1986); y 5.1-34.7 \%o en Yucatán (Andrade 1996). En su conjunto, esos trabajos explican la distribución espacial de $C$. sapidus en la Bahía de Chetumal, en los ámbitos de salinidad registrados en las estaciones de muestreo.

En la Bahía de Chetumal, C. sapidus presentó preferencia por las salinidades de $12.73 \pm 1.6 \%$; estos resultados concuerdan con los de Rocha-Ramírez et al. (1992) y Andrade (1996), reforzando el hecho de que la salinidad es un factor importante en la distribución espacial de $C$. sapidus.

El comportamiento temporal de C. sapidus en la Bahía de Chetumal, muestra que la abundancia durante la temporada de nortes es menor en comparación con la de lluvias y secas, siendo muy similar a lo observado por Buchanan y Stoner (1988) en Laguna Joyuda, Puerto Rico, donde la abundancia de C. sapidus, se incrementó durante la temporada de secas con una tendencia a disminuir al final de la temporada de lluvias. Moncada y Gómez (1980), informan que durante el invierno, las hembras de $C$. sapidus se sitúan a la entrada de la laguna de Tuna de Saza, Cuba; mientras que los machos se encuentran en aguas más internas y cercanas a la costa a una profundidad menor. Lo que fortalece lo citado por Buchanan y Stoner (1988), que en estuarios con variabilidad estacional en temperatura y salinidad, la abundancia relativa de esta especie podría exhibir fuertes diferencias espaciales y estacionales.

Las jaibas viven debajo de las rocas y otros objetos (Granados et al. 1995); sin embargo, $C$. sapidus no se asocia a algún sustrato específico (Raz-Guzmán y Sánchez 1996). En Jamaica se han encontrado en fondos fangosos (Norse 1972); entre 41 y $60 \%$ de arena y profundidades superiores a $9 \mathrm{~m}$ (Schaffner y Díaz 1988); en fondos de arena-fango y fangosos de Laguna Joyuda, Puerto Rico (Buchanan y Stoner 1988); en profundidades promedio entre $0.6-18.3 \mathrm{~m}$ y en sedimentos con porcentajes promedio de grava-arena entre 1-80\% (Rothschild et al. 1992). De Jesús-Navarrete et al. (2000), informaron profundidades de $1-5 \mathrm{~m}$, sedimentos con más del $90 \%$ de arenas y un porcentaje de materia orgánica de 2.6-20.3\%, en 43 estaciones de la Bahía de Chetumal, resultados similares a los registrados en este estudio. Esto puede indicar, que C. sapidus se encuentra en fondos de diferente textura y profundidades variables.

Los mayores valores de materia orgánica encontradas principalmente en las estaciones del sector A y C, son próximas a la costa, por lo que el aporte de materia orgánica provendría de manglares, vegetación circundante y ribereña (Manzanero 1999), y a la acumulación a largo plazo, debido a la suspensión de materiales autóctonos producidos en las cercanías, como detritos de hojas de pasto marino y algas (De Jesús-Navarrete et al. 2000). Lo que permitiría considerarlos como sitios potenciales de fuentes de alimentación para las jaibas.

Los machos de C. sapidus tienden a permanecer en áreas de más baja salinidad que las hembras (Churchill 1919, Schaffner y Díaz 1988, Rothschild et al. 1992, Andrade 1996, Guillory et al. 1996). Hines et al. (1987) encontraron que hay una división de hábitat por sexo, donde la proporción de sexos de los organismos adultos difiere espacialmente con respecto a la salinidad y temporalmente respecto al pico de apareamiento y migración de las hembras a zonas de mayor salinidad (Williams et al. 1990). En este estudio, no se encontraron hembras ovígeras y esto puede explicarse por las características mesohalinas de la Bahía de Chetumal y del ciclo de vida de C. sapidus. RománContreras (1986b) mencionó que la carencia de hembras ovígeras de Callinectes en la Laguna de Términos, Campeche, fue debido a que el muestreo se realizó en áreas abiertas, donde la influencia de aguas fluviales y marinas ya mezcladas no permiten valores altos de salinidad. Después de la inseminación, las hembras migran hacia el mar a áreas de alta salinidad (euhalinas) cerca de la boca de los estuarios, para desovar (Van Engel 1958, Tankersley et al. 1998). 
Las diferencias de abundancia espacial y temporal de jaibas del grupo II, pueden ser debido a que las jaibas grandes pueden elegir áreas deseables en términos de parámetros abióticos y suministros alimenticios (Buchanan y Stoner 1988). Rodríguez (1982), señala que en la zona camaronera del Golfo de Guacanayabo, Cuba, C. sapidus presenta una distribución fuertemente costera con un gradiente de abundancia en el sentido de disminuir a medida de que se alejan de la costa. La presencia de jaibas del grupo I en las estaciones del sector $\mathrm{A}$, puede deberse a que los jóvenes de $C$. sapidus tienden a moverse a regiones someras y menos salinas de los estuarios, para proveerse de alimento y refugio durante la fase de crecimiento rápido (Churchill 1919, Adkins 1972, Williams 1974, Hines et al. 1987). En Ría Celestún, Yucatán, la frecuencia de los organismos jóvenes de ambos sexos fue mayor en la zona interna (Andrade 1996). En Laguna Joyuda, Puerto Rico, la gran abundancia de jóvenes cerca de la entrada, puede ser el resultado de la migración de los jóvenes (Buchanan y Stoner 1988). La presencia de mayor abundancia de jóvenes en dos temporadas puede ser un indicio de reclutamiento. En la Bahía de Chetumal, las jaibas del grupo 0, de C. sapidus fueron escasas, lo que puede deberse a dos razones: la primera es que el arte de pesca empleado no sea eficiente para capturar individuos pequeños, y la segunda es que en la Bahía de Chetumal, las áreas de pastos que puedan brindar refugio y alimentación son limitadas, por lo que es posible que el área de manglar existente (no estudiada por nosotros) pueda satisfacer estas necesidades. Las jaibas pequeñas sobreviven mejor donde hay pocos depredadores y mejor protección a pesar de las condiciones ambientales variables (Buchanan y Stoner 1988).

La estructura por talla de C. sapidus mostró un patrón espacial y temporal consistente, lo que sugiere una estructura de la población definida. La CPUE mostró una correlación positiva con la temperatura y la salinidad, lo que hace suponer que los factores biológicos así como los factores físicos influyen en la distribución y abundancia de las jaibas (Buchanan y Stoner 1988).

Los patrones de distribución espacial parecen estar relacionados con las características de la salinidad, protección, alimentación y maduración; mientras que los patrones de distribución temporal pueden estar vinculados con la temperatura y salinidad, así como el reclutamiento y los eventos reproductivos.

\section{AGRADECIMIENTOS}

A Carmen Rosas y José Oliva por su valiosa colaboración en el trabajo de campo. Al pescador Daniel Robelo por su ayuda durante el trabajo de campo. A Julio Espinoza, Manuel Elías y Lourdes Vásquez, por comentarios que mejoraron sustancialmente el manuscrito. Este proyecto fue financiado por el Programa de Apoyo de Tesis de Maestría (PATM), Beca CONACYT No. 162709 y la Secretaría de Desarrollo Económico del Gobierno del Estado de Quintana Roo.

\section{RESUMEN}

Para determinar los patrones de distribución espacial y temporal de Callinectes sapidus, se realizaron muestreos durante las temporadas climáticas de nortes (enerofebrero), secas (mayo-junio) y lluvias (agosto-septiembre de 2002), en 30 estaciones de muestreo de la Bahía de Chetumal, agrupados en los sectores A (14 estaciones), B (ocho estaciones) y C (ocho estaciones). Para recolectar las jaibas se colocaron en cada sitio de muestreo dos transectos paralelos a la costa, con tres trampas cada uno, separadas $30 \mathrm{~m}$ una de otra. Se recolecciónron 1031 ejemplares de C. sapidus. La CPUE difirió espacial y temporalmente. La mayor CPUE fue encontrada en el sector C, con 1.3 ind trampa ${ }^{-1}$, y en la temporada climática de lluvias, con 1.1 ind trampa ${ }^{-1}$. La población estuvo compuesta predominantemente de individuos machos. La relación macho: hembra fue 15:1. La CPUE de machos y de adultos (grupo II) fue significativamente diferente entre sectores y entre temporadas climáticas. Ambos presentaron resultados similares, registrando la mayor CPUE en el sector C (1.2 ind trampa $\left.{ }^{-1}\right)$ y en la temporada climática de lluvias (1.1 ind trampa ${ }^{-1}$ ). La CPUE de hembras y de jóvenes del grupo I fue escasa durante el período de muestreo, mientras que la de jóvenes del grupo 0, fue nula. La mayor frecuencia relativa entre los sectores y las temporadas climáticas se 
observó en los intervalos de talla (AC) de 130-139 mm y 140-149 mm. C. sapidus se presentó en los sedimentos arenosos de la Bahía de Chetumal. La correlación producto momento de Pearson mostró relaciones significativas entre la CPUE de $C$. sapidus con la temperatura, salinidad y el oxígeno disuelto. Los patrones de distribución espacial y temporal mostrados por C. sapidus en la Bahía de Chetumal parecen estar relacionados con las características de salinidad, temperatura, protección, alimentación, reclutamiento y los eventos reproductivos.

Palabras clave: Caribe, Bahía de Chetumal, jaiba azul, Callinectes sapidus, distribución.

\section{REFERENCIAS}

Adkins, G. 1972. A study of the blue crab fishery in Louisiana. La. Wildl. Fish. Comm., Oyster, Water Bottoms and Seafood Div. Tech. Bull. No. 3. Baton Rouge, La. 57 p.

Andrade, M. 1996. Aspectos sobre la biología y ecología de las jaibas del género Callinectes de la laguna "Ría Celestún", Yucatán, México. Tesis de maestría, Centro de Investigación y de Estudios Avanzados, Mérida, México. 95 p.

Arreguín-Sánchez, S.F. 1976. Notas preliminares sobre las jaibas (Portunidae, Callinectes spp.) en las lagunas de Mandinga, Ver., Méx. p. 159-171. In Mem. Rec. Pesq. Cost. Méx. 23-25 Nov. INP, Veracruz, México.

Buchanan, B.A. \& W.A. Stoner. 1988. Distributional patterns of blue crabs (Callinectes sp.) in a tropical estuarine lagoon. Estuaries 11: 231-239.

Churchill, E.P., Jr. 1919. Life history of the blue crab. Bull. Bur. Fish. 36: 95-128.

Criales, M.M., C. Yeung, F. Amaya, A.C. López, D.L. Jones \& W.J. Richards. 2002. Larval supply of fishes, shrimps, and crabs into the nursery ground of the Ciénaga Grande de Santa Marta, Colombian Caribbean. Caribbean J. Sci. 38: 52-65.

Dean, W.E. 1974. Determination of carbonate and organic matter in calcareous sediments and sedimentary rocks by loss on ignition: comparison with other methods. Sediment. Petrol. 14: 242-248.

De Jesús-Navarrete, A., J.J. Oliva, V.V. Beltrán \& N. Quintero. 2000. Distribución de los sedimentos en la Bahía de Chetumal, Quintana Roo, México. Hidrobiológica 10: 61-67.

De la Lanza, E.G. 1986. Materia orgánica en los sedimentos del sistema lagunar Huizache y Caimanero:
Importancia, comportamiento y significado en modelos de predicción. An. Inst. Cienc. del Mar y Limnol. UNAM, México 13: 251-286.

Folk, R.L. 1969. Petrología de las rocas sedimentarias. Inst. Geol. UNAM, México DF, México. 399 p.

García-Montes, J.F., L.A. Soto \& A. Gracia. 1988. Cangrejos Portúnidos del Suroeste del Golfo de México: aspectos pesqueros y ecológicos. An. Inst. Cienc. del Mar y Limnol. UNAM 15: 135-150.

Gasca, R. \& I. Castellanos. 1993. Zooplancton de la Bahía de Chetumal, Mar Caribe, México. Rev. Biol. Trop. 41: 619-625.

Granados, B.A., J.L. Palma \& M.P. Pérez. 1995. Las jaibas del género Callinectes en la zona costera de Tabasco. Div. Acad. Cienc. Biol. 1: 5-13.

Guillory, V., P. Prejean, M. Bourgeois, J. Burdon \& J. Merrell. 1996. A biological and fisheries profile of the blue crab, Callinectes sapidus. La. Dep. Wildl. Fish., Fish. Manage. Plan Ser. No. 8, Part 1. 210 p.

Hernández, B.D.U., \& F. Pérez. 1991. Flora planctónica y producción primaria, p. 79-84. In T. Camarena-Luhrs \& S. Salazar-Vallejo (eds.). Estudios ecológicos preliminares de la zona sur de Quintana Roo. CIQRO, Quintana Roo, México.

Hines, A.H., R.N. Lipcius \& M. Haddon. 1987. Population dynamics and habitat partitioning by size, sex, and molt stage of blue crabs Callinectes sapidus in a subestuary of central Chesapeake Bay. Mar. Ecol. Prog. Ser. 36: 55-64.

Manzanero, R.E. 1999. Incidencia del tiempo de salado de la carnada en el rendimiento y estructura poblacional de las jaibas Callinectes spp. en la pesquería artesanal de Celestún, Yucatán, México. Tesis de licenciatura, Universidad Autónoma de Campeche, Campeche, México. 95 p.

Markham, J.C., F.E. Donath-Hernández, J.L. VillalobosHiart \& A.C. Díaz-Barriga. 1987. Notes of the shallow-water marine crustacean of the Caribbean coast of Quintana Roo, México. Anal. Inst. Biol. UNAM 61: 405-446.

Millikin, M.R. \& A.B. Williams. 1984. Synopsis of biological data on the blue crab, Callinectes sapi$d u s$ Rathbun. Food and Agriculture Organization Fisheries. Synopsis 138: 51-131.

Moncada, F.G. \& O. Gómez. 1980. Algunos aspectos biológicos de tres especies del género Callinectes (Crustacea-Decapoda). Rev. Cub. Inv. Pesq. 5:1-35. 
Morales-Vela, B., D. Olivera-Gómez \& P. Ramírez. 1996. Conservación de los manatíes en la región del Caribe de México y Belice. In Informe anual de Consejo Nacional de Ciencia y Tecnología. Centro de Investigaciones de Quintana Roo, Quintana Roo, México. 50 p.

Norse, E.A. 1972. Preliminary study of six Jamaican blue crabs, genus Callinectes (Decapoda: Portunidae). Atoll. Res. Bull. 152: 5.

Norse, E.A. 1977. Aspects of the zoogeographic distribution of Callinectes (Decapoda: Portunidae). Bull. Mar. Sci. 27: 440-447.

Norse, E.A. 1978. An experimental gradient analysis: Hyposalinity as an 'upstress' distributional determinant for Caribbean portunid crabs. Biol. Bull. 155: 586-598.

Olivera-Gómez, L.D. 2002. Asociación entre características del hábitat y la distribución y abundancia del manatí antillano (Trichechus manatus manatus) en el norte de la bahía de Chetumal, p. 61-66. In F.J. Rosado-May, R. Romero \& A. De Jesús-Navarrete (eds.). Contribuciones de la ciencia al manejo costero integrado de la bahía de Chetumal y su área de influencia. Universidad de Quintana Roo, Quintana Roo, México.

Ramos, P.J.L., A.A. Granados \& O. Lárraga. 1998. La pesquería de las jaibas del género Callinectes (Decapoda: Portunidae) en el suroeste del Golfo de México. Univ. Cienc. 14: 65-78.

Raz-Guzmán, A. \& A. Sánchez. 1996. Catálogo ilustrado de cangrejos braquiuros (Crustacea) de la laguna de Tamiahua, Veracruz, México. Inst. Biol. UNAM. México. 52 p.

Raz-Guzmán, A., A. Sánchez, L. Soto \& F. Álvarez. 1986. Catálogo ilustrado de cangrejos braquiuros y anomuros de la laguna de Términos, Campeche (Crustacea: Brachyura: Anomura). Inst. Biol. UNAM 57: 383.

Rocha-Ramírez, A., S. Cházaro-Olvera \& P.M. MuellerMeier. 1992. Ecología del género Callinectes (Brachyura: Portunidae) en seis cuerpos de aguas costeros del estado de Veracruz, México. An. Inst. Cienc. del Mar y Limnol. UNAM 19: 33-41.

Rodríguez, M.J. 1982. Posibilidades de aprovechamiento de la jaiba azul (Callinectes sapidus, Rathbun) de la fauna de acompañamiento de la pesquería de camarón de manzanillo. Rev. Cub. Inv. Pesq. 7:46-52.
Román-Contreras, R. 1986a. Comportamiento nictemeral de crustáceos decápodos en la boca de Estero Pargo, Laguna de Términos, Campeche, México. Anal. Inst. Cienc. del Mar y Limnol. UNAM 13: 149-158.

Román-Contreras, R. 1986b. Análisis de la población de Callinectes spp. (Decapoda: Portunidae) en el sector occidental de la Laguna de Términos, Campeche, México. Anal. Inst. Cienc. del Mar y Limnol. UNAM 13: 315-322.

Rosas, V.C. \& E. Lázaro-Chávez. 1986. Efecto de las variaciones de salinidad sobre la tasa respiratoria de dos especies de jaibas: Callinectes sapidus y Callinectes rathbunae, en la laguna de Tamiahua, Veracruz, México. Rev. Invest. Mar. 7: 71-79.

Rosas, V.C. \& A. Sánchez. 1994. Fisiología de la adaptación de los crustáceos decápodos al ambiente lagunar estuarino, p. 305-332. In G. de la Lanza \& C. Cáceres. (eds.) Lagunas costeras y el litoral mexicano. Universidad Autónoma de Baja California Sur, Baja California, México.

Rothschild, B.J., J.S. Ault, E.V. Patrick, C.I. Zhang, S.G. Smith, H. Li, T. Maurer, B. Daugherty, S. Endo \& R.N. Mc Garvey. 1992. Assessment of the Chesapeake Bay blue crab stock. In Final report to the Maryland Department of Natural Resources, the Chesapeake Bay Stock Assessment Committee and the National Oceanic and Atmospheric Administration, Washington DC, EEUU. 200 p.

Schaffner, L.C. \& R.J. Diaz. 1988. Distribution and abundance of overwintering blue crabs, Callinectes sapidus, in the lower Chesapeake Bay. Estuaries 11: 68-72.

Tankersley, R.A., M.G. Weiber, M.A. Sigala \& K.A. Kachurak. 1998. Migratory behavior of ovigerous blue crabs Callinectes sapidus: evidence for selective tidal-stream transport. Biol. Bull. 195: 168-173.

Van Engel, W.A. 1958. The blue crab and its fishery in Chesapeake Bay. Part I. Reproduction, early development, growth and migration. Commer. Fish. Rev. 20: 6-17.

Williams, A.B. 1974. The swimming crabs of the genus Callinectes (Decapoda: Portunidae). Fish. Bull. 72: 685-789.

Williams, H.A., L.D. Coen \& M.S. Stoelting. 1990. Seasonal abundance, distribution and habitat selection of jóvenes Callinectes sapidus (Rathbun) in the Northern Gulf of Mexico. J. Exp. Mar. Biol. Ecol. 140: 107-119. 
